\title{
Vitamin D und Infektanfälligkeit
}

\author{
Vitamin D and Susceptibility to Infection
}

Autoren

Institute
A. Zittermann' ${ }^{1}$ S. Pilz², W. März ${ }^{3,4,5}$

Die Institute sind am Ende des Artikels gelistet.

\author{
Schlüsselwörter \\ - Vitamin D \\ - 25-Hydroxyvitamin D \\ - Infektionen \\ - Immunabwehr \\ Keywords \\ 0 vitamin D \\ - 25-hydroxyvitamin D \\ - infection \\ - immune defense
}

\section{Bibliografie}

Dol http://dx.doi.org/ 10.1055/s-0035-1552694 Aktuel Ernahrungsmed 2015; 40: $240-246$

(c) Georg Thieme Verlag KC Stuttgart · New York ISSN 0341-0501

\section{Korrespondenzadresse}

Prof. Dr. Armin Zittermann Klinik für Thorax- und Kardiovaskularchirurgie Herz- und Diabeteszentrum NRW

Georgstraße 11

32545 Bad Oeynhausen

Tel.: +49 (0) 5731 97-1912

Fax: +49 (0) 5731 97-2020

azittermann@hdz-nrw.de

\section{Zusammenfassung \\ $\nabla$}

Bereits in früheren Jahrhunderten sind Lebertran und UVB-Strahlung, von denen man heutzutage weiß, dass sie die effizientesten Vitamin-D-Quellen für den Menschen sind, erfolgreich zur Behandlung der Tuberkulose eingesetzt worden.

Der vorliegende Artikel gibt einen Überblick über die Funktionen von Vitamin D im Immunsystem sowie über die Datenlage zum Einfluss von Vitamin D auf Infektionen.

Einige randomisierte kontrollierte Studien, wenn auch nicht alle, unterstützen die Hypothese, dass Vitamin D das Tuberkuloserisiko reduzieren kann. Eine Metaanalyse randomisierter kontrollierter Studien hat ergeben, dass durch Supplementierung mit Vitamin D die Anfälligkeit gegenüber Infektionen des oberen Respirationstrakts im Mittel um etwa ein Drittel gesenkt werden kann. Hierbei ist offensichtlich die tägliche Gabe effektiver als die Bolusgabe von Vitamin D. Bekanntlich treten in Europa und Nordamerika insbesondere im Winterhalbjahr häufig Infektionen des oberen Respirationstrakts auf. Prospektive Kohortenstudien lassen vermuten, dass das Infektionsrisiko bei 25-Hydroxyvitamin-D-Konzentrationen unter $25 \mathrm{nmol} / \mathrm{l}(10 \mathrm{ng} / \mathrm{ml})$ am höchsten, bei Konzentrationen über $75 \mathrm{nmol} / 1(30 \mathrm{ng} / \mathrm{ml})$ am geringsten ist. Bei einer Reihe weiterer Infektionserkrankungen ist denkbar, dass die Vitamin-D-Versorgung das Erkrankungsrisiko beeinflusst, die Datenlage ist aber für eine abschließende Bewertung noch nicht ausreichend.

In der Gesamtschau sind die Ergebnisse zur Reduzierung der Infektanfälligkeit des oberen Respirationstrakts durch Vitamin D wahrscheinlich. In Übereinstimmung mit Empfehlungen verschiedener Ernährungsgesellschaften schließen wir, dass bei unzureichender Vitamin-D-Versorgung (z.B. 25-Hydroxyvitamin-D-Spiegel $<50 \mathrm{nmol} / \mathrm{l}$ oder $20 \mathrm{ng} / \mathrm{ml}$ ) Vitamin D am besten täglich supple-

\section{Abstract \\ $\nabla$}

It has long been known that both cod liver oil and solar UVB radiation, which are nowadays known as the most potent vitamin $\mathrm{D}$ sources, can be successfully used to treat tuberculosis.

The present article gives an overview about the effects of vitamin D on the immune system. Moreover, current evidence regarding vitamin D and infections is summarized.

Some, but not all randomized controlled trials support the hypothesis that vitamin D may reduce the risk of tuberculosis. A meta-analysis of randomized controlled trials has demonstrated that vitamin D supplements can reduce susceptibility against upper respiratory tract infections by approximately one third. Daily supplements seem to be more effective than bolus administration of vitamin D. It is well-known that upper respiratory tract infections are most prevalent in Europe and North America in the winter half-year. Prospective cohort studies suggest that the risk of infection is highest in those individuals with deficient circulating 25-hydroxyvitamin D levels $(<25$ $\mathrm{nmol} / \mathrm{l}$ ) and is lowest in those with 25-hydroxyvitamin D levels ( $>75 \mathrm{nmol} / \mathrm{l})$. In a number of other infectious disorders it seems to be possible that vitamin D status may influence disease risk. For a substantiated appraisal, however, available data is insufficient at present.

In total, results regarding risk reduction of upper respiratory tract infection by vitamin $\mathrm{D}$ can be considered likely. In line with recommendations of different nutrition societies it is concluded that in case of insufficient vitamin D status (e.g. 25-hydroxyvitamin D levels $<50 \mathrm{nmol} / 1$ or $<20$ $\mathrm{ng} / \mathrm{ml}$ ), (supplemental) oral daily vitamin $\mathrm{D}$ intake should be ensued. Intake should be in the order of 1000 international units. 
mentiert werden sollte. Die Zufuhr sollte in der Größenordnung von 1000 internationalen Einheiten liegen.

\section{Allgemeines}

Vitamin D nimmt unter den Vitaminen eine Sonderstellung ein, da der Mensch grundsätzlich in der Lage ist, es selbst zu synthetisieren ( $\bullet$ Abb.1). Voraussetzung hierfür ist jedoch, dass die Haut in ausreichendem Maße der ultravioletten Sonnenstrahlung der Wellenlänge 290-315 nm (UVB-Strahlung) ausgesetzt ist. Nennenswerte Mengen an Vitamin D kommen natürlicherweise auch in einigen Lebensmitteln vor (insbesondere Fettfische wie Aal, Lachs und Hering sowie Lebertran). Da der Mensch grundsätzlich Vitamin D selbst produzieren kann, betrachten viele Kliniker Vitamin D eher als Prohormon denn als Vitamin. Bei einer Reihe von Bevölkerungsgruppen ist die Synthese in der Haut jedoch eingeschränkt oder fehlt fast vollständig. Hierzu zählen Büroarbeiter, Heimbewohner und dunkelhäutige Personen, die in nördlichen Breitengraden leben, sowie Frauen, die traditionellen religiösen Kleidervorschriften folgen. Aufgrund der zunehmenden Zahl der meisten der oben genannten Gruppen ist es sehr wahrscheinlich, dass in Zukunft der Vitamincharakter von Vitamin D, d. h. die orale Zufuhr, in den Vordergrund des Interesses rücken wird.

Der Vitamin-D-Status wird am besten durch die Erfassung des im Blut zirkulierenden 25-Hydroxyvitamin D [25(OH)D] bestimmt. Hierbei handelt es sich um das erste Hydroxylierungsprodukt von Vitamin D, das in der Leber gebildet wird. Das nordamerikanische Institute of Medicine (IOM) [1] hat Blutspiegel an 25OHD von $50-125 \mathrm{nmol} / \mathrm{l}(20-50 \mathrm{ng} / \mathrm{ml})$ als adäquat eingestuft $(1 \mathrm{nmol} /$ $\mathrm{l}=0,4 \mathrm{ng} / \mathrm{ml}$ ), Werte zwischen 30 und 49,99 (12 und 19,99 $\mathrm{ng} / \mathrm{ml}$ ) als inadäquat, Werte unter $30 \mathrm{nmol} / \mathrm{l}(12 \mathrm{ng} / \mathrm{ml})$ als defizitär und Werte über $125 \mathrm{nmol} / \mathrm{l}(50 \mathrm{ng} / \mathrm{ml})$ als potenziell gesundheitsschädlich. Als Kriterium für eine adäquate Versorgung wird hierbei ausschließlich die Knochengesundheit angeführt. Als Argument für potenziell schädliche Wirkungen ab $125 \mathrm{nmol} / \mathrm{l}$ werden vor allem die Ergebnisse verschiedener prospektiver Kohortenstudien auf Tumorinzidenz, Myokardinfarkt und Gesamtmortalität genannt. Die Endocrine Society [2] sieht allerdings eine adäquate Versorgung erst ab $75 \mathrm{nmol} / \mathrm{l}$ gewährleistet. Neben positiven Effekten auf den Bewegungsapparat (Muskeln, Knochen), werden explizit auch potenziell positive extraskeletale Gesundheitseffekte durch Vitamin D angeführt. Ein oberer Grenzwert für eine adäquate Versorgung wird nicht genannt.

Die aktive Hormonform ist das 1,25-Dihydroxyvitamin D [1,25 $(\mathrm{OH})_{2} \mathrm{D}$ ], die vor allem in der Niere, aber auch in verschiedenen extrarenalen Geweben aus dem 25(OH)D gebildet wird. Die Konzentration des $1,25(\mathrm{OH})_{2} \mathrm{D}$ im Blut wird in der Regel sehr fein reguliert und ist deshalb weniger aussagekräftig zur Beurteilung der Versorgungslage als die des 25(OH)D. Im Fall einer inadäquaten oder defizitären Vitamin-D-Versorgung ist jedoch auch die im Blut zirkulierende Menge an $1,25(\mathrm{OH})_{2} \mathrm{D}$ vermindert, und zwar abhängig von der Konzentration des 25(OH)D [3].

Um eine adäquate Vitamin-D-Versorgung zu gewährleisten, empfiehlt das IOM im ersten Lebensjahr 400 internationale Einheiten $(1 \mathrm{IE}=0,025 \mu \mathrm{g})$ sowie nach dem Säuglingsalter und bis zu einem Alter von 70 Jahren eine tägliche Vitamin-D-Zufuhr von 600 IE. Für Personen > 70 Jahre beträgt die tägliche Empfehlung 800 IE [1]. Die DACH-Referenzwerte für Erwachsene betragen für Personen mit fehlender Vitamin-D-Synthese in der Haut ebenfalls 800 IE [4]. Den oberen Grenzwert einer sicheren tägli-

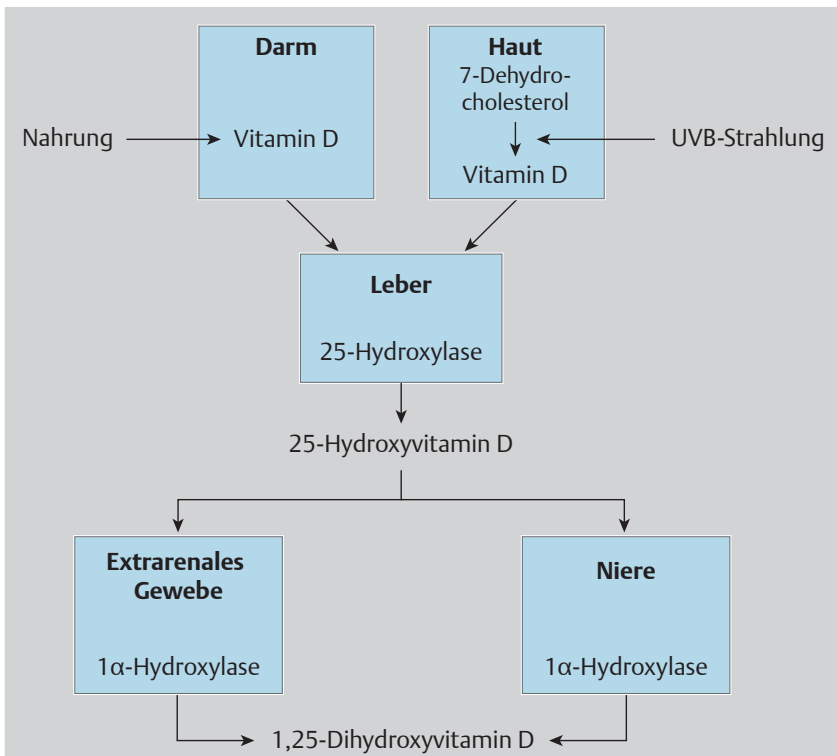

Abb. 1 Schematische Darstellung des Stoffwechsels von Vitamin D.

chen Vitamin-D-Zufuhr hat das IOM ebenso wie die europäische Behörde für Lebensmittelsicherheit bei $4000 \mathrm{IE}$ festgelegt.

Die zentrale Rolle von Vitamin D für den Kalzium- und Phosphathaushalt sowie für die Knochengesundheit ist gut bekannt. Es mehren sich jedoch die Hinweise, dass Vitamin D auch eine Reihe nichtklassischer Wirkungen im Stoffwechsel des Menschen entfaltet. Der vorliegende Artikel gibt einen Überblick über die aktuelle Datenlage bezüglich der Bedeutung von Vitamin D für das Immunsystem und die Infektanfälligkeit. Der Beitrag basiert auf einem Konsensus-Meeting zum Thema, das am 16. Mai 2014 in Frankfurt/M stattfand.

\section{Historie \\ $\nabla$}

Bereits in früheren Jahrhunderten sind Lebertran und UVBStrahlung, von denen man heutzutage weiß, dass sie am effektivsten zu einer adäquaten Vitamin-D-Versorgung des Menschen beitragen, erfolgreich zur Behandlung der Tuberkulose eingesetzt worden. Die Tuberkulose ist eine intrazelluläre Infektionskrankheit, die durch Mykobakterien verursacht wird, und vor allem die Lunge, aber prinzipiell auch jedes andere Organ betreffen kann. Im 19. und frühen 20. Jahrhundert war die Tuberkulose in Europa weit verbreitet und für ca. 25-50\% aller Todesfälle beim Erwachsenen verantwortlich. Im Jahr 1848 wurden in einer der ersten klinischen Studien mehr als 1000 Tuberkulosepatienten entweder lediglich pflegerisch betreut, da keine effektive Therapie bekannt war, oder die Patienten bekamen einen Löffel Lebertran 3-mal am Tag. Das Ergebnis war, dass sich lediglich bei 19\% der Patienten unter Lebertrangabe, jedoch bei 33\% der Patienten in der Kontrollgruppe die Krankheit verschlimmerte bzw. die Patienten starben [5]. Im Jahre 1903 erhielt Niels Finsen den Nobelpreis für Medizin und Physiologie für seine Theorie zur Heilung von Lupus vulgaris (Hauttuberkulose) mittels Fototherapie. Die Tuberkulose ist heutzutage weltweit immer noch die zweithäufigste Todesursache aller Infektionserkrankungen [6]. Um eine erhöhte Sterblichkeit zu vermeiden, sollten die Patienten mindestens 6 Monate lang mit Tuberkulosemedikamenten therapiert werden [7]. 


\section{Aktuelle Vitamin-D-Versorgungslage $\nabla$}

Säuglinge sind in Deutschland und Österreich adäquat mit Vitamin D versorgt, wenn sie die empfohlene Rachitisprophylaxe mit 400 - 500 IE Vitamin D pro Tag bekommen. Nach einer repräsentativen Erhebung des Robert Koch-Instituts [8] wird im zweiten Lebensjahr in Deutschland die Rachitisprophylaxe noch bei ca. einem Drittel der Kinder (meist bei denen, die im Winter geboren wurden) durchgeführt. In dieser Altersklasse weisen 7\% der deutschstämmigen Kinder und 10\% der Jungen bzw. 17\% der Mädchen mit Migrationshintergrund entsprechend der IOM-Klassifikation bereits eine mangelhafte Versorgung auf. Bei weiteren 24-28\% der 2-Jährigen ist die Versorgungslage inadäquat. Der Anteil der mangelhaft Versorgten steigt dann ab dem 3. Lebensjahr - wenn Vitamin-D-Supplemente nicht mehr flächendeckend eingenommen werden - in allen Altersgruppen auf ca. 15-17\% an. Eine Ausnahme bilden Mädchen mit Migrationshintergrund. Hier liegt der Anteil der mangelhaft Versorgten sogar bei über $30 \%$. Weitere $45 \%$ aller Kinder über 3 Jahren und aller Jugendlichen weisen eine inadäquate Versorgung auf. Repräsentative Erhebungen des Robert Koch-Instituts [9] haben gezeigt, dass bei ca. 15-17\% der erwachsenen Bevölkerung die 25 (OH)D-Konzentrationen im defizitären, und bei weiteren $40 \%$ im inadäquaten Bereich liegen. Bei Patienten ist die Situation generell noch ungünstiger: Blutuntersuchungen bei Hausarztpatienten, die zwischen Februar und Mai durchgeführt wurden [10], ergaben je nach Altersklasse einen Anteil an mangelhaft Versorgten zwischen $20 \%$ und $38 \%$. Jüngste umfangreiche Daten aus Norddeutschland [11] konnten die Ergebnisse im Wesentlichen bestätigen: Während im Sommer weniger als 10\% der Patienten eine mangelhafte Vitamin-D-Versorgung aufweisen, steigt dieser Anteil in den Wintermonaten auf bis zu $40 \%$ an. Besonders ungünstig ist die Versorgungslage bei Patienten, die sich einer Rehabilitationsmaßnahme unterziehen [12] sowie bei Heimbewohnern [13], von denen $67 \%$ bzw. 75\% eine defizitäre Vitamin-D-Versorgung aufweisen. Auch Übergewichtige haben in Deutschland 25 $(\mathrm{OH}) \mathrm{D}-$ Konzentrationen, die im Mittel nur knapp oberhalb der Obergrenze der Defizienz liegen [14]. Insgesamt verdeutlichen die Daten, dass Vitamin-D-Mangel weit verbreitet ist und somit optimale Vitamin-D-abhängige Stoffwechselprozesse häufig nicht gewährleistet sind.

\section{Vitamin-D-Wirkungen auf die Immunabwehr \\ $\nabla$}

Seit den 1980er-Jahren wurde zunehmend deutlich, dass Vitamin D insbesondere bei der unspezifischen Immunabwehr eine herausragende Rolle spielt. So findet man Vitamin-D-Rezeptoren unter anderem auf Monozyten. Diese Zellen differenzieren sich unter Einfluss von 1,25(OH) ${ }_{2} \mathrm{D}$ zu Makrophagen. Letztere stellen eine erste und wichtige unspezifische Verteidigungslinie des Immunsystems dar. Makrophagen besitzen selbst $1 \alpha$-Hydroxylaseaktivität und können somit bei Bedarf das aktive Hormon aus 25( $\mathrm{OH}) \mathrm{D}$ produzieren. Bei aktivierten Makrophagen ist die Aktivität dieses Enzyms stark erhöht [15]. Makrophagen können ebenso wie Epithelzellen verschiedene antimikrobielle Peptide (AMPs) wie Cathelicidin und Defensine produzieren, deren Synthese direkt (Cathelicidin) oder indirekt (Defensine) unter Kontrolle von 1,25( $\mathrm{OH})_{2} \mathrm{D}$ steht [16]. Diese AMPs können Viren, Pilze und Bakterien abtöten. In Makrophagen wird durch $1,25(\mathrm{OH})_{2} \mathrm{D}$ auch die Aktivität lysosomaler Enzyme gesteigert sowie die Synthese von reaktiven Sauerstoffspezies und die Produktion von
Stickstoffmonoxid (NO) erhöht, was letztlich zu einer vermehrten zytotoxischen Aktivität und Phagozytoserate führt [17]. Im Jahre 2006 gelang erstmals der Nachweis, dass Mycobacterium tuberculosis in menschlichen Makrophagen die Expression des Vitamin-D-Rezeptors sowie des $1 \alpha$-Hydroxylase-Gens steigert. Vitamin D wiederum steigert sehr effektiv auf der Transkriptionsebene die Synthese des (einzigen beim Menschen exprimierten) Cathelicidins (LL-37), wodurch intrazelluläre Mykobakterien abgetötet werden [18]. 1,25( $\mathrm{OH})_{2} \mathrm{D}$ induziert auch die Autophagie von Monozyten und schützt dadurch den Körper vor einer intrazellulären Infektion mit Mycobacterium tuberculosis. Diese Vitamin-D-Wirkungen beruht ebenfalls auf einer Steigerung der Cathelicidin-Produktion [19]. Sowohl bei Proben von Personen mit insuffizienten 25(OH)D-Spiegeln als auch bei Proben mit niedrigen $1,25(\mathrm{OH})_{2} \mathrm{D}$-Spiegeln, ist die Fähigkeit von Monozyten zur Cathelicidin-Produktion reduziert [19]. Vitamin D ist auch in der Lage, die Aktivität der natürlichen Killerzellen (NK) zu erhöhen [20]. Diese Zellen schützen den Körper vor Viren und anderen Krankheitserregern. Einige Viren sind in der Lage, Haupt-Histokompatibilitäts-Komplex (MHC) Klasse-I-Moleküle auf der Oberfläche ihrer Wirtszelle zu unterdrücken und sich damit der Zerstörung durch T-Lymphozyten zu entziehen. Allerdings macht der Verlust der MHC-Moleküle diese Zellen anfällig für NK-Zellvermittelte Apoptose. Die antivirale Wirkung von Vitamin D, die gegenüber Influenza-Infektionen vermutet wird, basiert vermutlich u.a. auch auf einer Verhinderung der Hämagglutination [21]. Darüber hinaus könnten die Krankheitssymptome einer Influenza-Infektion dadurch vermindert werden, dass Vitamin D die Synthese proinflammatorischer Zytokine supprimiert [21, 22]. Dagegen ist ein niedriger Vitamin-D-Status mit einer Aktivierung von Entzündungsprozessen assoziiert [23]. Vitamin D kann ebenfalls die spezifische Immunantwort beeinflussen indem es - induziert durch T-Helfer-Zellen vom Typ 2 - eine erhöhte Freisetzung von Immunoglobulin E sowie eine Eosinophilie fördert und dadurch extrazelluläre Infektionen durch Parasiten, Protozoen und Pilze bekämpft [24].

\section{Vitamin D und Tuberkulose}

$\nabla$

Während die Tuberkulose heutzutage in Afrika und Asien noch weit verbreitet ist, erkranken insgesamt nur noch wenige 1000 Patienten pro Jahr in den deutschsprachigen Ländern an Tuberkulose. Hierbei handelt es sich primär um Migranten. Es wird vermutet, dass sich die Migranten in ihren Herkunftsländern infizieren, die Erkrankung aber erst in Europa durch eine mangelhafte Vitamin-D-Versorgung ausbricht [25]. In $\bullet$ Tab. 1 sind verschiedene randomisierte kontrollierte Studien aufgeführt, die den Effekt von Vitamin D auf die Tuberkulose untersucht haben. Die Studien wurden in Asien $(n=4)$, Afrika $(n=1)$ und Großbritannien $(n=2)$ durchgeführt und in der Mehrzahl der Studien wurden hohe Bolusgaben an Vitamin D verabreicht. Der Tuberkulosenachweis erfolgte in verschiedenen Flüssigkeiten/Organen wie Sputum, Blut und Haut. Insgesamt sind die Ergebnisse heterogen. Einen Einfluss auf die Ergebnisse hatte möglicherweise die initiale Vitamin-D-Versorgung sowie die Dauer und die Dosis der Vitamin-D-Gabe. Auffallend ist, dass in den beiden Studien mit einer Hazard Ratio von 0,95 und 1,0 [28, 31] die initialen $25(\mathrm{OH})$ D-Spiegel entsprechend der IOM-Klassifikation bereits im adäquaten Bereich lagen. Zusätzlich lagen die 25(OH)D-Spiegel bei der Studie von Salahuddin et al. [28] zu Studienende mit 220 $\mathrm{nmol} / \mathrm{l}$ deutlich oberhalb des Wertes, der vom IOM als unbedenk- 
Tab. 1 Randomisierte kontrollierte Studien zu Vitamin D und Tuberkulose.

\begin{tabular}{|c|c|c|c|c|c|c|c|c|c|c|c|}
\hline \multirow[t]{2}{*}{$\begin{array}{l}\text { Autor } \\
\text { (Ref.) }\end{array}$} & \multirow[t]{2}{*}{$\begin{array}{l}\text { Publika- } \\
\text { tionsjahr }\end{array}$} & \multirow[t]{2}{*}{$\begin{array}{l}\mathrm{N} \\
\text { (total) }\end{array}$} & \multirow[t]{2}{*}{$\begin{array}{l}\text { Alter } \\
\text { (Jahre) }\end{array}$} & \multicolumn{2}{|c|}{$\begin{array}{l}250 \mathrm{HD}(\mathrm{nmol} / \mathrm{l}) \\
\text { initial }\end{array}$} & \multicolumn{2}{|c|}{$\begin{array}{l}\text { 250HD (nmol/l) } \\
\text { Studienende }\end{array}$} & \multirow[t]{2}{*}{$\begin{array}{l}\text { mittlere Vita- } \\
\text { min-D-Dosis } \\
\text { (IE) }\end{array}$} & \multirow[t]{2}{*}{ Dauer } & \multirow[t]{2}{*}{ Endpunkt } & \multirow[t]{2}{*}{$\begin{array}{l}\text { Ergebnisse } \\
\text { HR (95\%-KI) }\end{array}$} \\
\hline & & & & Vit. D & Plazebo & Vit. D & Plazebo & & & & \\
\hline $\begin{array}{l}\text { Nursyam } \\
{[26]}\end{array}$ & 2006 & 67 & 31 & n.b. & n.b. & n.b. & n.b. & 10000 täglich & 6 Wochen & $\begin{array}{l}\text { Sputum- } \\
\text { konversion }\end{array}$ & $0,68(0,52-0,89)$ \\
\hline $\begin{array}{l}\text { Marti- } \\
\text { neau [27] }\end{array}$ & 2007 & 192 & 38 & 35 & n.b. & 67 & n.b. & $\begin{array}{l}100000 \text { Ein- } \\
\text { maldosis }\end{array}$ & 6 Wochen $^{1}$ & Bluttest & $0,80(0,65-0,98)$ \\
\hline $\begin{array}{l}\text { Wejse } \\
{[28]}\end{array}$ & 2009 & 365 & 37 & 77 & 79 & 98 & 95 & $\begin{array}{l}100000 \\
\text { 4-monatlich }\end{array}$ & 12 Monate & $\begin{array}{l}\text { Sputum- } \\
\text { konversion }\end{array}$ & $1,0^{2}$ \\
\hline $\begin{array}{l}\text { Marti- } \\
\text { neau [29] }\end{array}$ & 2011 & 146 & 31 & 21 & 21 & 101 & 23 & 4-mal 100000 & 56 Tage & $\begin{array}{l}\text { Sputum- } \\
\text { konversion }\end{array}$ & $0,72(0,46-1,11)$ \\
\hline $\begin{array}{l}\text { Ganmaa } \\
{[30]}\end{array}$ & 2012 & 120 & 13 & 18 & 18 & 50 & 24 & 800 täglich & 6 Monate & $\begin{array}{l}\text { Tuberkulin- } \\
\text { hauttest }\end{array}$ & $0,41(0,16-1,09)$ \\
\hline $\begin{array}{l}\text { Salahud- } \\
\text { din [31] }\end{array}$ & 2013 & 259 & 28 & 52 & 58 & 220 & 50 & 2-mal 600000 & 12 Wochen & $\begin{array}{l}\text { Sputum- } \\
\text { konversion }\end{array}$ & $0,95(0,51-1,78)$ \\
\hline Ralph [32] & 2013 & 200 & 28 & n.b. & n.b. & n.b. & n.b. & $\begin{array}{l}50000 \text { monat- } \\
\text { lich }\end{array}$ & 8 Wochen & $\begin{array}{l}\text { Sputum- } \\
\text { konversion }\end{array}$ & $1,31(0,68-2,51)$ \\
\hline
\end{tabular}

$25 \mathrm{OHD}=25$-Hydroxyvitamin D; IE= internationale Einheiten; $n$. b. = nicht bestimmt; HR= Hazard Ratio

${ }^{1}$ Es handelt sich um die Beobachtungsdauer, bei allen anderen Studien handelt es sich um die Therapiedauer; ${ }^{2}$ zum Untersuchungsende war kein Erreger im Sputum mehr nachweisbar, weder in der Vitamin-D-Gruppe noch in der Plazebo-Gruppe

lich klassifiziert wird, sodass unerwünschte Effekte nicht grundsätzlich auszuschließen sind. In der Studie mit ungünstiger Hazard Ratio für Vitamin D $(>1,0)$ [32] wurden die 25(OH)D-Werte nicht gemessen. Bei allen anderen Studien war die Hazard Ratio zumindest im Mittel deutlich zugunsten der Vitamin-D-Gabe reduziert, wobei in 2 Studien [26, 27] die Ergebnisse signifikant waren. Die Vitamin-D-Gabe war bei allen Studien, bei denen entsprechende 25(OH)D-Messwerte vorlagen, ausreichend um die Spiegel bis zum Studienende auf mindestens $50 \mathrm{nmol} / \mathrm{l}$ anzuheben.

\section{Vitamin D und Infekte des oberen Respirationstrakts}

$\nabla$

Studien zum Einfluss von Vitamin D auf Infekte des Respirationstrakts sind schwierig, weil Dauer und Ausmaß der Infekte nicht immer gut objektiv erfassbar sind. Vor diesem Hintergrund ist eine finnische Studie [33] positiv hervorzuheben: Bei 800 Rekruten, d.h. einer homogenen Gruppe von grundsätzlich gesunden jungen Männern, wurde das Fernbleiben vom Dienst aufgrund von Infektionen des Respirationstrakts untersucht. Es stellte sich heraus, dass Rekruten mit 25(OH)D-Konzentrationen $<40 \mathrm{nmol} / \mathrm{l}$ $(16 \mathrm{ng} / \mathrm{ml})$ signifikant häufiger dienstunfähig waren als Rekruten mit besserem Vitamin-D-Status. Basierend auf diesen Daten wurde eine randomisierte kontrollierte Folgestudie im Winterhalbjahr (Oktober bis März) durchgeführt [34], bei der die Rekruten entweder 400 IE Vitamin D oder ein Placebo bekamen. In der Vitamin-D-Gruppe war die durchschnittliche Dauer der Fehltage tendenziell niedriger als in der Placebogruppe (im Mittel 2,2 vs. 3,0 Tage). Darüber hinaus lag der Prozentsatz an Rekruten ohne Fehlzeiten in der Vitamin-D-Gruppe signifikant niedriger als in der Placebogruppe (35,7\% vs. 51,3\%). Eine im Jahr 2013 publizierte Metaanalyse von randomisierten kontrollierten Studien hat den Einfluss einer Vitamin-D-Einnahme auf das Risiko für Infektionen des Respirationstrakts systematisch analysiert [35]. Es wurden 11 Studien in die Analyse eingeschlossen, deren Charakteristika und Ergebnisse in $\odot$ Tab. 2 dargestellt sind. Hiervon waren 8 Studien mit Gesunden und 3 Studien mit Patienten durch- geführt worden, wobei die Studienteilnehmer in 6 Studien Kinder und in 5 Studien Erwachsene waren. Insgesamt konnte das Infektionsrisiko durch Vitamin-D-Gabe um ein Drittel gesenkt werden. Interessanterweise zeigte in der zusätzlichen Subgruppenanalyse nur ein Faktor signifikante Unterschiede zwischen den Subgruppen, und zwar war die tägliche Vitamin-D-Gabe mit einem signifikant besseren Ergebnis assoziiert als eine (hoch dosierte) Bolusgabe an Vitamin D. Die Ergebnisse sind aus 2 Gründen von besonderem Interesse: Erstens liefern die Daten Beweise dafür, dass Vitamin D neben seinen Effekten auf den Bewegungsapparat weitere wichtige Funktionen besitzt, die für den Menschen von klinischer Relevanz sind. Zweitens zeigen die Daten, dass der Vitamin-D-Versorgung nicht nur im Säuglingsalter und bei Senioren Beachtung geschenkt werden muss, sondern auch bei ansonsten gesunden Kindern und Erwachsenen. Im Winter ist nicht nur eine defizitäre/inadäquate Vitamin-D-Versorgung in der Bevölkerung weit verbreitet, auch Infektionen des Respirationstrakts sind häufig.

In einer weiteren Studie, die im Jahr 2013 von Rees et al. publiziert wurde ( $\bullet$ Tab.2), führte die Vitamin-D-Einnahme lediglich tendenziell zu einer geringeren Inzidenz an Infektionen des oberen Respirationstrakts im Winter. Wenn allerdings die Infektionen während des gesamten Jahres berücksichtigt wurden, war die Inzidenzrate gegenüber der Kontrollgruppe signifikant geringer (relatives Risiko: 0,91; 95\%-Konfidenzintervall: 0,83-0,99). Bereits vor einigen Jahren ergab die Auswertung eines umfangreichen US-Datensatzes [47], dass Infektionen des oberen Respirationstrakts erwartungsgemäß saisonal stark variieren, wobei der Häufigkeitsgipfel im Winter liegt. Defizitär versorgte Personen $(<25 \mathrm{nmol} / \mathrm{l}$ oder $<10 \mathrm{ng} / \mathrm{ml})$ wiesen jedoch stets ein höheres Risiko als adäquat $(>75 \mathrm{nmol} / \mathrm{l}$ oder $>30 \mathrm{ng} / \mathrm{ml})$ mit Vitamin D versorgte Personen auf.

Da virale Infektionen des Respirationstrakts häufig durch bakterielle Infektionen überlagert werden, sind die Ergebnisse einer Post-hoc-Analyse der RECORD-Studie [48], einer prospektiven, randomisierten Studie mit 5300 Teilnehmern, die entweder täglich 800 IE Vitamin D oder Placebo über 2 - 5 Jahre erhielten, interessant: In der Vitamin-D-Gruppe bestand nicht nur eine Ten- 
Tab. 2 Randomisierte, kontrollierte Studien zu Vitamin D und Infektionen des Respirationstrakts.

\begin{tabular}{|c|c|c|c|c|c|c|c|c|c|c|}
\hline \multirow[t]{2}{*}{ Autor (Ref.) } & \multirow[t]{2}{*}{$\begin{array}{l}\text { Publika- } \\
\text { tionsjahr }\end{array}$} & \multirow[t]{2}{*}{$\mathbf{N}$ (total) } & \multirow[t]{2}{*}{$\begin{array}{l}\text { Alter } \\
\text { (Jahre) }\end{array}$} & \multicolumn{2}{|c|}{$\begin{array}{l}250 \mathrm{HD}(\mathrm{nmol} / \mathrm{l}) \\
\text { initial }\end{array}$} & \multicolumn{2}{|c|}{$\begin{array}{l}250 \mathrm{OD}(\mathrm{nmol} / \mathrm{l}) \\
\text { Studienende }\end{array}$} & \multirow[t]{2}{*}{$\begin{array}{l}\text { mittlere Vita- } \\
\text { min-D-Dosis (IE) }\end{array}$} & \multirow[t]{2}{*}{ Dauer } & \multirow[t]{2}{*}{$\begin{array}{l}\text { Ergebnisse } \\
\text { HR (95\%-KI) }\end{array}$} \\
\hline & & & & Vit. D & Plazebo & Vit. D & Plazebo & & & \\
\hline Aloia [36] & 2007 & 208 & 60,6 & 46,9 & 43,0 & 86,9 & 43,0 & $800 / 2000$ täglich & 3 Jahre & $0,25(0,11-0,58)$ \\
\hline Li-Ng [37] & 2009 & 148 & 58,7 & 64,3 & 63,0 & 88,5 & 60,9 & 2000 täglich & 3 Monate & $0,79(0,41-1,54)$ \\
\hline Laaksi [34] & 2010 & 164 & $\begin{array}{l}\text { Männer, } \\
\text { jung }\end{array}$ & 78,7 & 74,4 & 72,0 & 51,0 & 400 täglich & 6 Monate & $0,67(0,38-1,17)$ \\
\hline $\begin{array}{l}\text { Manaseki- } \\
\text { Holland [38] }\end{array}$ & 2010 & 453 & 1,2 & n.b. & n.b. & n.b. & n.b. & $\begin{array}{l}100000 \text { (Einmal- } \\
\text { dosis) }\end{array}$ & 3 Wochen & $0,60(0,41-0,80)$ \\
\hline $\begin{array}{l}\text { Urashima } \\
\text { [39] }\end{array}$ & 2010 & 334 & 10,2 & n.b. & n.b. & n.b. & n.b. & 1200 täglich & 4 Monate & $0,53(0,28-0,99)$ \\
\hline Majak [40] & 2011 & 48 & 11,5 & 90,0 & 88,0 & 94,0 & 80,0 & 500 täglich & 6 Monate & $0,24(0,06-0,90)$ \\
\hline $\begin{array}{l}\text { Bergman } \\
{[41]}\end{array}$ & 2012 & 124 & 53,1 & 51,5 & 46,9 & 117,4 & 44,0 & 4000 täglich & 12 Monate & $0,48(0,25-0,91)$ \\
\hline $\begin{array}{l}\text { Camargo } \\
{[42]}\end{array}$ & 2012 & 244 & 9,97 & 17,5 & 17,0 & 47,3 & 18,0 & 300 täglich & 7 Wochen & $0,49(0,31-0,79)$ \\
\hline Jorde [43] & 2012 & 569 & 63,0 & n.b. & n.b. & n.b. & n.b. & $3344^{*}$ & 12 Monate & $0,93(0,52-1,64)$ \\
\hline $\begin{array}{l}\text { Manaseki- } \\
\text { Holland [44] }\end{array}$ & 2012 & 3046 & 0,8 & n.b. & n.b. & $\#$ & $\S$ & $\begin{array}{l}100000 \text { quartals- } \\
\text { weise }\end{array}$ & 18 Monate & $1,04(0,92-1,19)$ \\
\hline $\begin{array}{l}\text { Murdoch } \\
\text { [45] }\end{array}$ & 2012 & 322 & 47,5 & 75,5 & 70,0 & 122,5 & 55,0 & $\begin{array}{l}100000 \text { monat- } \\
\text { lich }^{+}\end{array}$ & 18 Monate & $0,92(0,62-1,37)$ \\
\hline Rees [46] & 2013 & 759 & 58,1 & 62,0 & 63,1 & n.b. & n.b. & 1000 täglich & 3-5 Jahre & $0,93(0,79-1,10)$ \\
\hline
\end{tabular}

$25 \mathrm{OHD}=25$-Hydroxyvitamin D; IE= internationale Einheiten; n. b. = nicht bestimmt; HR= Hazard Ratio; KI=Konfidenzintervall. Die Vitamin-D-Dosen der Einzelstudien waren wie folgt: 2000, 2800 und $6800 \mathrm{IU} /$ täglich, 20000 und $40000 \mathrm{IU} /$ wöchentlich sowie 100 000/monatlich, zweimonatlich oder dreimonatlich

* es wurden mehrere Studien eingeschlossen; * signifikant höher als in der Plazebogruppe; ${ }^{\S}$ signifikant niedriger als in der Vitamin-D-Gruppe; ${ }^{+}$initiale Dosis: 200000 IE

denz für eine geringere Neigung für Infektionen, sondern auch der Antibiotikaeinsatz war tendenziell geringer. In einer anderen Post-hoc-Analyse einer Vitamin-D-Studie wurde ein altersabhängiger Effekt von Vitamin D auf die Antibiotika-Einnahme beobachtet [49]: Während Vitamin-D-Supplemente im Vergleich zur Kontrollgruppe die Antibiotikaeinnahme bei Personen unter 70 Jahren nicht reduzierten, benötigten Personen ab 70 Jahren in der Kontrollgruppe deutlich mehr Antibiotika als in der VitaminD-Gruppe. Insgesamt lassen es die Daten somit als wahrscheinlich erscheinen, dass eine ausreichende Vitamin-D-Versorgung auch für die Infektabwehr notwendig ist.

\section{Sonstige Infektionserkrankungen}

Bei einer Reihe weiterer Infektionserkrankungen liegen Hinweise vor, dass die Vitamin-D-Versorgung das Erkrankungsrisiko beeinflusst. So ist ein Vitamin-D-Defizit mit einem erhöhten Sepsisrisiko assoziiert [50]. Des Weiteren konnte durch tägliche oder wöchentliche Vitamin-D-Gabe eine latente Reaktivierung des Epstein-Barr-Virus verhindert werden, und zwar umso besser je höher der 25(OH)D-Spiegel unter Vitamin-D-Gabe lag [51]. Interventionsstudien lassen auch vermuten, dass der Therapieerfolg bei Hepatitis-C-Infektionen durch gleichzeitige Vitamin-D-Gabe verbessert wird [52]. Bei vielen anderen wichtigen Infektionserkrankungen wie beispielsweise bei Infektionen des Urogenitaltrakts und Wundinfektionen, ist die Datenlage zum Einfluss von Vitamin D auf den Krankheitsverlauf dagegen bisher noch unzureichend. Zukünftige Studien müssen daher zeigen, bei welchen Infektionserkrankungen Vitamin D eine präventive Wirkung entfalten kann, ab welchen Konzentrationen eine Substitution sinnvoll ist, ob die Wirkung einer Vitamin-D-Substitution bei verschiedenen Bevölkerungsgruppen gleich ist (gesunde, unterschiedliche Patientengruppen), und ob Vitamin D auch therapeutisch, und wenn ja, in welcher Dosierung, eingesetzt werden soll.

\section{Fazit}

Vitamin-D-Mangel ist in Deutschland weit verbreitet. Die vorliegenden Daten deuten darauf hin, dass die Vitamin-D-Versorgung Einfluss auf das Risiko und den Verlauf von Infektionskrankheiten nimmt. Eine Optimierung der Versorgung ist daher auch unter diesem Aspekt anzustreben. Insbesondere im Winter ist ein erhöhter oraler Bedarf aufgrund der in dieser Jahreszeit geringeren UVB-Strahlung der Sonne gegeben. Zur Prävention von Infekten und zur Optimierung der unspezifischen Immunabwehr ist es sinnvoll und sicher, täglich ca. $1000 \mathrm{IE}$ an Vitamin D einzunehmen. Dabei ist es wichtig, zu Beginn des Herbstes mit der Supplementierung zu beginnen, um einen adäquaten Vitamin-D-Spiegel im Winter zu gewährleisten. Bei Personen mit erhöhtem Risiko für einen Vitamin-D-Mangel ist eine Supplementierung auch ganzjährig durchzuführen. Dazu zählen vor allem im Büro tätige Menschen und immobile Senioren.

\section{Danksagung}

Das Konsensus-Meeting wurde finanziell von der Fa. Merck, Darmstadt, unterstützt. Die Fa. Merck hatte keinen Einfluss auf das Ergebnis des Konsensus-Meetings.

\section{Interessenkonflikt}

$\nabla$

Die Autoren geben an, dass kein Interessenkonflikt besteht.

\section{Institute}

${ }^{1}$ Klinik für Thorax- und Kardiovaskularchirurgie, Herz- und Diabeteszentrum NRW, Ruhr-Universität Bochum, Deutschland

2 Abteilung für Endokrinologie und Stoffwechsel, Medizinische Universität Graz, Österreich

${ }^{3}$ Klinisches Institut für Medizinische und Chemische Labordiagnostik, Medizinische Universität Graz, Österreich 
${ }^{4}$ Medizinische Klinik V (Nephrologie, Hypertensiologie, Rheumatologie, Endokrinologie, Diabetologie), Medizinische Fakultät Mannheim, Universität Heidelberg, Deutschland

${ }^{5}$ Synlab Akademie für Ärztliche Fortbildung, Mannheim und Synlab Services $\mathrm{GmbH}$, Augsburg, Deutschland

\section{Literatur}

1 Ross AC, Manson JE, Abrams SA et al. The 2011 report on dietary reference intakes for calcium and vitamin $D$ from the Institute of Medicine: what clinicians need to know. J Clin Endocrinol Metab 2011; 96: $53-58$

2 Holick MF, Binkley NC, Bischoff-Ferrari HA et al. Endocrine Society. Evaluation, treatment, and prevention of vitamin D deficiency: an Endocrine Society clinical practice guideline. J Clin Endocrinol Metab 2011; 96: $1911-1930$

3 Docio S, Riancho JA, Pérez A et al. Seasonal deficiency of vitamin D in children: a potential target for osteoporosis-preventing strategies? J Bone Miner Res 1998; 13: 544-548

4 Deutsche Gesellschaft für Ernährung, Österreichische Gesellschaft für Ernährung, Schweizerische Gesellschaft für Ernährungsforschung Schweizerische Vereinigung für Ernährung. D-A-CH-Referenzwerte für die Nährstoffzufuhr. 1. Auflage, 5., korrigierter Nachdruck 2013. Neustadt an der Weinstraße: Neuer Umschau Buchverlag; 2012

5 Kupferschmidt $K$. Uncertain verdict as vitamin D goes on trial. Science 2012; 337: 1476 - 1478

6 WHO. Globaltuberculosiscontrol: WHO report. Accessed March 24, 2015 at http://www.who.int/iris/handle/10665/75938

7 Tiemersma EW, van der Werf MJ, Borgdorff MW et al. Natural history of tuberculosis: duration and fatality of untreated pulmonary tuberculosis in HIV negative patients: a systematic review. PLoS One 2011; 6: e17601

8 Hintzpeter B, Scheidt-Nave C, Müller MJ et al. Higher prevalence of vitamin $D$ deficiency is associated with immigrant background among children and adolescents in Germany. J Nutr 2008; 138: 1482 - 1490

9 Hintzpeter B, Mensink GB, Thierfelder $W$ et al. Vitamin D status and health correlates among German adults. Eur J Clin Nutr 2008; 62: $1079-1089$

10 Zittermann A. The estimated benefits of vitamin D for Germany. Mol Nutr Food Res 2010; 54: 1164-1171

11 Kramer J, Diehl A, Lehnert H. Epidemiological study on the dimension of vitamin D deficiency in North Germany. Dtsch Med Wochenschr 2014; 139: 470 - 475 [in German]

12 Schilling S. Epidemic vitamin D deficiency among patients in an elderly care rehabilitation facility. Dtsch Arztebl Int 2012; 109: 33 - 38

13 Pilz S, Dobnig H, Tomaschitz A et al. Low 25-hydroxyvitamin D is associated with increased mortality in female nursing home residents. J Clin Endocrinol Metab 2012; 97: E653-657

14 Zittermann A, Frisch S, Berthold HK et al. Vitamin D supplementation enhances the beneficial effects of weight loss on cardiovascular disease risk markers. Am J Clin Nutr 2009; 89: 1321 - 1327

15 Zittermann A. Vitamin D in preventive medicine: are we ignoring the evidence? Br J Nutr 2003; 89: 552 - 572

16 Zasloff M. Fighting infections with vitamin D. Nat Med 2006; 12: $388-$ 390

17 Sly LM, Lopez M, Nauseef WM et al. 1alpha, 25-Dihydroxyvitamin D3induced monocyte antimycobacterial activity is regulated by phosphatidylinositol 3-kinase and mediated by the NADPH-dependent phagocyte oxidase. J Biol Chem 2001; 276: 35482 - 35493

18 Liu PT, Stenger S, Li H et al. Toll-like receptor triggering of a vitamin Dmediated human antimicrobial response. Science 2006; 311: 1770 1773

19 Dini C, Bianchi A. The potential role of vitamin D for prevention and treatment of tuberculosis and infectious diseases. Ann Ist Super Sanita 2012; 48: 319-327

20 Zittermann A. Vitamin D in der Präventivmedizin. 2. Auflage. Bremen: UNI-MED Verlag; 2012

21 Urashima M, Segawa T, Okazaki M et al. Randomized trial of vitamin D supplementation to prevent seasonal influenza A in schoolchildren. Am J Clin Nutr 2010; 91: 1255-1260

22 Grant WB, Giovannucci E. The possible roles of solar ultraviolet-B radiation and vitamin $\mathrm{D}$ in reducing case-fatality rates from the 1918-1919 influenza pandemic in the United States. Dermatoendocrinol 2009; $1: 215-219$
23 Murr C, Pilz S, Grammer TB et al. Vitamin D deficiency parallels inflammation and immune activation, the Ludwigshafen Risk and Cardiovascular Health (LURIC) study. Clin Chem Lab Med 2012; 50: 2205 - 2212

24 Kearns MD, Alvarez JA, Seidel $N$ et al. Impact of Vitamin D on Infectious Disease: A Systematic Review of Controlled Trials. Am J Med Sci 2015; 349: $245-262$

25 Chan TYK. Vitamin D deficiency and susceptibility to tuberculosis. Calcif Tissue Int 2000; 66: 476-478

26 Nursyam EW, Amin Z, Rumende CM. The effect of vitamin D as supplementary treatment in patients with moderately advanced pulmonary tuberculous lesion. Acta Med Indones 2006; 38: 3-5

27 Martineau AR, Wilkinson RJ, Wilkinson KA et al. A single dose of vitamin D enhances immunity to mycobacteria. Am J Respir Crit Care Med 2007; 176: 208-213

28 Wejse C, Gomes VF, Rabna P et al. Vitamin D as supplementary treatment for tuberculosis: a double-blind, randomized, placebo-controlled trial. Am J Respir Crit Care Med 2009; 179: 843 - 850

29 Martineau AR, Timms PM, Bothamley GH et al. High-dose vitamin D(3) during intensive-phase antimicrobial treatment of pulmonary tuberculosis: a double-blind randomised controlled trial. Lancet 2011; 377: $242-250$

30 Ganmaa D, Giovannucci E, Bloom BR et al. Vitamin D, tuberculin skin test conversion, and latent tuberculosis in Mongolian school-age children: a randomized, double-blind, placebo-controlled feasibility trial. Am J Clin Nutr 2012; 96: 391 - 396

31 Salahuddin N, Ali F, Hasan Z et al. Vitamin D accelerates clinical recovery from tuberculosis: results of the SUCCINCT Study [Supplementary Cholecalciferol in recovery from tuberculosis]. A randomized, placebocontrolled, clinical trial of vitamin D supplementation in patients with pulmonary tuberculosis'. BMC Infect Dis 2013; 13: 22

32 Ralph AP, Waramori G, Pontororing GJ et al. L-arginine and vitamin D adjunctive therapies in pulmonary tuberculosis: a randomised, double-blind, placebo-controlled trial. PLoS One 2013; 8: e70032

33 Laaksi I, Ruohola JP, Tuohimaa P et al. An association of serum vitamin D concentrations $<40 \mathrm{nmol} / \mathrm{L}$ with acute respiratory tract infection in young Finnish men. Am J Clin Nutr 2007; 86: 714-717

34 Laaksi I, Ruohola JP, Mattila V et al. Vitamin D supplementation for the prevention of acute respiratory tract infection: a randomized, doubleblinded trial among young Finnish men. J Infect Dis 2010; 202: 809 814

35 Bergman P, Lindh AU, Björkhem-Bergman $L$ et al. Vitamin D and Respiratory Tract Infections: A Systematic Review and Meta-Analysis of Randomized Controlled Trials. PLoS One 2013; 8: e65835

36 Aloia JF, Li-Ng M. Epidemic influenza and vitamin D. Epidemiol Infect 2007; 135: 1095 - 1096

$37 \mathrm{Li}-\mathrm{Ng} \mathrm{M}$, Aloia JF, Pollack $S$ et al. A randomized controlled trial of vitamin D3 supplementation for the prevention of symptomatic upper respiratory tract infections. Epidemiol Infect 2009; 137: 1396-1404

38 Manaseki-Holland S, Qader G, Isaq Masher M et al. Effects of vitamin D supplementation to children diagnosed with pneumonia in Kabul: a randomised controlled trial. Trop Med Int Health 2010; 15: 1148 1155

39 Urashima M, Segawa T, Okazaki M et al. Randomized trial of vitamin D supplementation to prevent seasonal influenza A in schoolchildren. Am J Clin Nutr 2010; 91: 1255-1260

40 Majak P, Olszowiec-Chlebna M, Smejda K et al. Vitamin D supplementation in children may prevent asthma exacerbation triggered by acute respiratory infection. J Allergy Clin Immunol 2011; 127: 1294-1296

41 Bergman P, Norlin AC, Hansen S et al. Vitamin D3 supplementation in patients with frequent respiratory tract infections: a randomised and double-blind intervention study. BMJ Open 2012; 2. pii: e001663

42 Camargo CA Jr, Ganmaa D, Frazier AL et al. Randomized trial of vitamin D supplementation and risk of acute respiratory infection in Mongolia. Pediatrics 2012; 130: e561-567

43 Jorde $R$, Witham $M$, Janssens $W$ et al. Vitamin D supplementation did not prevent influenza-like illness as diagnosed retrospectively by questionnaires in subjects participating in randomized clinical trials. Scand J Infect Dis 2012; 44: 126-132

44 Manaseki-Holland S, Maroof Z, Bruce J et al. Effect on the incidence of pneumonia of vitamin D supplementation by quarterly bolus dose to infants in Kabul: a randomised controlled superiority trial. Lancet 2012; 379: 1419-1427

45 Murdoch DR, Slow S, Chambers ST et al. Effect of vitamin D3 supplementation on upper respiratory tract infections in healthy adults: the VIDARIS randomized controlled trial. JAMA 2012; 308: 1333-1339 
46 Rees JR, Hendricks $K$, Barry EL et al. Vitamin D3 supplementation and upper respiratory tract infections in a randomized, controlled trial. Clin Infect Dis 2013; 57: 1384-1392

47 Ginde AA, Mansbach JM, Camargo CA. Association between serum 25hydroxyvitamin D level and upper respiratory tract infection in the Third National Health and Nutrition Examination Survey. Arch Intern Med 2009; 169: 384-390

48 Avenell A, Cook JA, Maclennan GS et al. Vitamin D supplementation to prevent infections: a sub-study of a randomised placebo-controlled trial in older people (RECORD trial, ISRCTN 51647438). Age Ageing 2007; 36: 574-577
49 Tran B, Armstrong BK, Ebeling PR et al. Effect of vitamin D supplementation on antibiotic use: a randomized controlled trial. Am J Clin Nutr 2014; 99: $156-161$

50 Han JE, Ziegler TR. Vitamin d supplementation in sepsis and critical illness: where are we now? Am J Respir Crit Care Med 2014; 190: $483-$ 485

51 Zwart SR, Mehta SK, Ploutz-Snyder R et al. Response to vitamin D supplementation during Antarctic winter is related to BMI, and supplementation can mitigate Epstein-Barr Virus Reactivation. J Nutr 2011; 141: $692-697$

52 Villar LM, Del Campo JA, Ranchal I et al. Association between vitamin D and hepatitis C virus infection: a meta-analysis. World J Gastroenterol 2013; 19: $5917-5924$ 